\title{
Analytical modelling of drain-current characteristics of AlGaN/GaN HFETs with full incorporation of steady-state velocity overshoot
}

\author{
Alireza Loghmany and Pouya Valizadeh \\ Department of Electrical and Computer Engineering, Concordia University, Montreal, Canada \\ E-mail: pouya@ece.concordia.ca
}

Received 1 October 2010, in final form 4 February 2011

Published 10 March 2011

Online at stacks.iop.org/JPhysD/44/125102

\begin{abstract}
An analytical model for drain-current characteristics of $\mathrm{AlGaN} / \mathrm{GaN}$ heterostructure field-effect transistors (HFETs), with incorporation of steady-state velocity overshoot and the inflection points in the electronic drift-transport characteristics, is presented. Manifestations of these transport characteristics are usually neglected in modelling the drain current of III-V HFETs. However, significance of these features in $\mathrm{AlGaN} / \mathrm{GaN}$ material system, compared with other III-V technologies, requires re-evaluation of this policy. Although, for the current state of the art these features are partially masked by the parasitic features such as the drain and source contact resistance, through further improvement in the device fabrication technology they will deserve further attention. The wide peak and pronounced inflection points in the transport characteristics of $\mathrm{AlGaN} / \mathrm{GaN}$ heterojunctions are modelled through considering a drift-diffusion channel rather than a drift-only transport channel. Simulation results are compared with a non-diffusion type channel that has been so far assumed in modelling the drain current of these devices. Results of the presented model indicate the role of drift-velocity overshoot in further increasing the maximum drain current of $\mathrm{AlGaN} / \mathrm{GaN}$ HFETs, as the contact resistance is reduced. Comparisons with the experimental measurements are also provided.
\end{abstract}

\section{Introduction}

Polar III-nitride semiconductors, including AlGaN/GaN heterojunction, due to their superb two-dimensional electron gas (2DEG) concentration, high peak and saturation electron velocity, and wide bandgap have been the target of much attention over the past two decades. With improvements in material growth techniques [1,2], device fabrication [3] and long-term reliability [4], an increasing need is being felt for the development of models capable of accurately incorporating particular features of this material system.

The study of Bhapkar et al reveals that in addition to the steady-state velocity overshoot there exists a pronounced kink in the low electric-field region of the drift velocity versus electric-field characteristics (i.e. $v-E$ ) of $\mathrm{GaN}$ [5]. The existence of the inflection points attributed to this kink and the large width of the overshoot pattern in conjunction with the large electric fields conventionally applied to this wide-bandgap semiconductor make the modelling of electronic devices fabricated in this technology different from those of other III-V semiconductors.

In this work, the influence of the particular $v-E$ characteristics of $\mathrm{GaN}$ on the drain current-voltage characteristics of an $\mathrm{AlGaN} / \mathrm{GaN}$ heterostructure field-effect transistor (HFET) is modelled. Presence of these features dictates the formation of electron bunching across the 2DEG channel, especially at high drain voltages. The impact of this non-zero gradient in electron concentration is modelled by considering the diffusion-current component in regions of the channel with electric-field values corresponding to the moderate electric-field inflection points and high electricfield negative differential mobility. In the design of 
AlGaN/GaN, HFETs in order to take advantage of the wide bandgap of the semiconductor in extending the breakdown voltage, traditionally the total channel length (i.e. source-drain spacing) amounts to a few micrometres. As a result, nonsteady-state velocity overshoot and ballistic transport are not considered in this drift-diffusion treatment of the transport problem. Also, as is traditionally the case for long-channel FETs, in order to keep the model analytically manageable discussions of the energy relaxation time constants are avoided.

The degree of complexity of the drift-transport model employed in the transport problem is a determinant factor to the possibility of yielding either an analytical or a numerical solution. So far, several transport models have been proposed for $\mathrm{AlGaN} / \mathrm{GaN}$ heterojunctions [6-8]. Simpler transport models, such as Ridley's saturating transport model [7], yield easily solvable analytical models for drain currentvoltage characteristics of HFETs [9]. However, more complex transport models, despite the burden of numerical solutions, are worth studying for better projection of the impact of transport properties on future-generation devices. To incorporate further details of the electronic drift transport of the $\mathrm{AlGaN} / \mathrm{GaN}$ 2DEG, such as inflection points in the moderate electricfield region and the steady-state velocity overshoot under higher electric fields, a transport model previously reported by Polyakov and Schwierz [8] has been employed in this work. Due to the complexity of this transport model, the analytical model only yields to numerical solutions.

The influence of parasitic contact resistance on draincurrent characteristics of an $\mathrm{AlGaN} / \mathrm{GaN}$ HFET is also investigated. While, an ongoing research effort is being pursued to improve the Ohmic contact quality of AlGaN/GaN HFETs [10,11], the current state of the art of this technology still shows masking of some of the transport features in the drain-current characteristics. In this study, the effectiveness of the present model in unveiling these features is revealed.

In section 2 details of the implementation of the proposed model is presented. In section 3 results of the application of the proposed model to AlGaN/GaN HFETs are presented with respect to the variation of source and drain parasitic contact resistance and gate length. These results have been compared with the outcomes of the application of two simpler transport models to the proposed model. Conclusions are presented in section 4 .

\section{Description of the model}

So far, drift-transport characteristics of $\mathrm{GaN}$ channels, predicted by Monte Carlo simulations [5, 12-14], to different degrees of approximation have been presented by a few analytical expressions [6-8]. Among these models, Ridley's transport model provides the most manageable framework for development of an analytical model for drain current of HFETs [7]. According to Ridley's drift-transport model, electron drift velocity (i.e. $v(E)$ ) is determined in the form of a two-section model (in which $v_{\mathrm{s}}$ and $E_{\mathrm{s}}$ are the saturation velocity and electric field at the onset of velocity saturation, and $v_{0}$ is the knee velocity):

$$
\begin{gathered}
v(E)= \begin{cases}v_{0} E \frac{E+E_{1}}{E_{1}\left(E+E_{0}\right)} & E \leqslant E_{\mathrm{s}} \\
v_{\mathrm{s}} & E>E_{\mathrm{s}}\end{cases} \\
\text { where } E_{0}=\frac{v_{0}}{\mu_{0}} \quad E_{1}=\frac{v_{0}}{\mu_{1}}
\end{gathered}
$$

where $\mu_{0}=1000 \mathrm{~cm}^{2} \mathrm{~V}^{-1} \mathrm{~s}^{-1}, \mu_{1}=50 \mathrm{~cm}^{2} \mathrm{~V}^{-1} \mathrm{~s}^{-1}, v_{\mathrm{s}}=$ $1.4 \times 10^{7} \mathrm{~cm} \mathrm{~s}^{-1}, v_{0}=7.2 \times 10^{6} \mathrm{~cm} \mathrm{~s}^{-1}$.

Despite the lack of consideration for drift-velocity overshoot, this transport model has been observed to provide sufficient accuracy in calculating the drain current of the stateof-the-art AlGaN/GaN HFET, which possess a considerable value of contact resistance [9]. In this work, two more accurate transport models have been used to investigate the degree of approximation in the drain current-voltage characteristics inherited from (1). The first of these two transport models has been previously investigated in modelling GaN MESFETs [8]. This model is capable of accurately following the inflection points and drift-velocity overshoot pattern predicted by Monte Carlo simulations [5]. Drift velocity according to this transport model is given by

$$
v(E)=\frac{\mu_{0} E+\mu_{1} E\left(E / E_{0}\right)^{\alpha}+v_{\mathrm{sat}}\left(E / E_{1}\right)^{\beta}}{1+\left(E / E_{0}\right)^{\alpha}+\left(E / E_{1}\right)^{\beta}}
$$

where $\mu_{0}=720 \mathrm{~cm}^{2} \mathrm{~V}^{-1} \mathrm{~s}^{-1}, \mu_{1}=234 \mathrm{~cm}^{2} \mathrm{~V}^{-1} \mathrm{~s}^{-1}, E_{0}=$ $3.36 \times 10^{4} \mathrm{~V} \mathrm{~cm}^{-1}, E_{1}=9.8 \times 10^{4} \mathrm{~V} \mathrm{~cm}^{-1}, \alpha=2.44, \beta=$ 7.45 .

The second drift-transport model, to which (1) is compared, presents the drift velocity as

$$
v(x)=\frac{\mu_{0} E(x)+v_{\mathrm{s}}\left(E(x) / E_{1}\right)^{5}}{1+\left(E(x) / E_{0}\right)^{5}}
$$

where $\mu_{0}=260 \mathrm{~cm}^{2} \mathrm{~V}^{-1} \mathrm{~s}^{-1}, v_{\mathrm{s}}=2.1 \times 10^{7} \mathrm{~cm} \mathrm{~s}^{-1}, E_{0}=$ $15.9 \times 10^{4} \mathrm{~V} \mathrm{~cm}^{-1}, E_{1}=17.2 \times 10^{4} \mathrm{~V} \mathrm{~cm}^{-1}$.

The parameters in (1) have been chosen to provide the maximum similarity to the saturation velocity predicted in [14] and also to agree with the low-field electron mobility of the AlGaN/GaN HFET reported in section 3. Parameters of (2) were also selected with regards to this low-field electron mobility, in addition to the inflection point, velocity overshoot and the saturation velocity reported in [14]. Parameters of (3) were tuned to provide the best match with (2) in the peak-tosaturation velocity range. These three $v-E$ characteristics are shown in figure 1.

Figure 2 illustrates the cross-section of an AlGaN/GaN HFET. In modelling the AlGaN/GaN HFET, the ungated drain and source access-regions are seen as ungated HFETs connected in series with a self-aligned gated HFET. These regions are identified in figure 2 . While due to the low electric fields of the source-access region, the current-voltage characteristics of this ungated part of the channel can be explained by an Ohmic characteristic, the current saturation in the drain-access region should be taken into account. The influence of parasitic contact resistance of source and drain is also included in this model through incorporation of their Ohmic values (i.e. $R_{\mathrm{S}}$ and $R_{\mathrm{D}}$, respectively). Table 1 shows the device parameters used for simulation, unless identified otherwise. 


\subsection{Drain-current simulation of the self-aligned HFET}

The approach adopted in modelling the drain-current characteristics of a self-aligned HFET involves splitting of the channel into two regions:

(1) Region I is referred to as the linear-region of the channel, along which the electric field is smaller than the threshold electric field corresponding to the drift-velocity overshoot (i.e. $E_{v \text {-max }}$ ).

(2) Region II is referred to as the saturation region of the channel, along which the electric field is beyond $E_{v \text {-max }}$.

Under low drain voltages, as the maximum electric field is smaller than $E_{v \text {-max }}$, region I will cover the entire length of the channel. With increasing the drain voltage, region II starts to form at the drain edge and expands at the cost of retraction of region I. Regions I and II are treated in sections 2.1.1 and 2.1.2, respectively. While in these sub-sections equations only with the assumption of the transport model of (2) are provided, for the other two transport models (i.e. (1) and (3)), counterpart equations can be easily developed.

2.1.1. Linear-region characteristics (i.e. $E<E_{v \text {-max }}$ ). In this region, by neglecting the gate leakage and leakage through

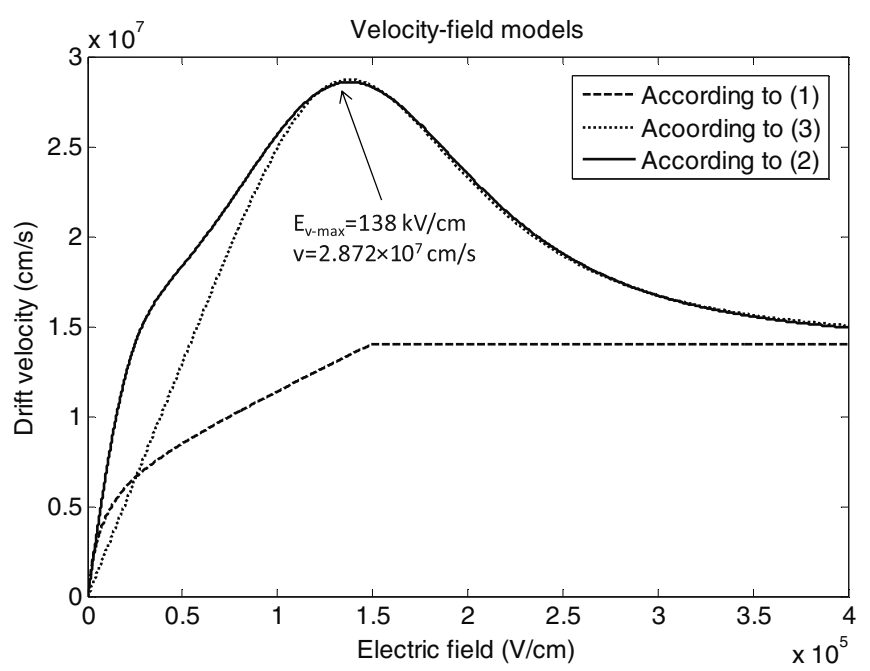

Figure 1. Drift velocity versus electric-field characteristics according to the three drift-transport models. the buffer layer, the drain-current density at any given point along the channel can be written as

$$
J=q n_{\mathrm{s}}(y) v(E(y)) .
$$

In which, in the presence of a gate bias (i.e. $V_{\mathrm{G}}$ ), the $2 \mathrm{DEG}$ density at the $\mathrm{AlGaN} / \mathrm{GaN}$ heterointerface can be expressed in terms of threshold voltage $V_{\mathrm{T}}$ :

$$
q n_{\mathrm{s}}(y)=C_{\mathrm{ch}}\left(V_{\mathrm{G}}-V_{\mathrm{T}}-V(y)\right)
$$

$V(y)$ is the channel potential and $C_{\mathrm{ch}}$ is the gate capacitance per unit area:

$$
C_{\mathrm{ch}}=\frac{\varepsilon_{0} \varepsilon_{\mathrm{AlGaN}}}{d+\Delta d}
$$

where $\varepsilon_{\mathrm{AlGaN}}$ is the relative dielectric constant of the barrier, $d$ is the barrier thickness and $\Delta d$ is the effective thickness of the 2DEG, which is typically $2-4 \mathrm{~nm}$ for an AlGaN/GaN 2DEG [15].

By substituting (2), (5) and (6) in (4), the following relationship between the drain-current density, longitudinal electric field (i.e. $E$ ) and channel potential at any given point in region I will be resulted:

$$
\begin{aligned}
(1+ & \left.\left(\frac{E}{E_{0}}\right)^{\alpha}+\left(\frac{E}{E_{1}}\right)^{\beta}\right) J=\left(\mu_{0} \cdot E+\mu_{1} E\left(\frac{E}{E_{0}}\right)^{\alpha}\right. \\
& \left.+v_{\mathrm{sat}}\left(\frac{E}{E_{1}}\right)^{\beta}\right) C_{\mathrm{ch}}\left(V_{\mathrm{GT}}-V(y)\right)
\end{aligned}
$$

in which $V_{\mathrm{GT}}$ is the effective gate voltage (i.e. $V_{\mathrm{G}}-V_{\mathrm{T}}$ ). Replacing $E(y)$ with the spatial derivative of channel potential in (7) leads to the following expression in terms of channel potential and drain-current density:

$$
\begin{gathered}
\frac{1}{E_{0}^{\alpha}}\left(J-C_{\mathrm{ch}}\left(V_{\mathrm{GT}}-V(y)\right) \mu_{1} \frac{\mathrm{d} V}{\mathrm{~d} y}\right)\left(\frac{\mathrm{d} V}{\mathrm{~d} y}\right)^{\alpha} \\
+\frac{1}{E_{1}^{\beta}}\left(J-C_{\mathrm{ch}}\left(V_{\mathrm{GT}}-V(y)\right) v_{\mathrm{sat}}\right)\left(\frac{\mathrm{d} V}{\mathrm{~d} y}\right)^{\beta} \\
-C_{\mathrm{ch}}\left(V_{\mathrm{GT}}-V(y)\right) \mu_{0} \frac{\mathrm{d} V}{\mathrm{~d} y}+J=0 .
\end{gathered}
$$

Equation (8) requires numerical solution and can be solved using the finite difference technique. In the case of the application of drift-transport models of (1) and (3), such a relationship will be analytically manageable.

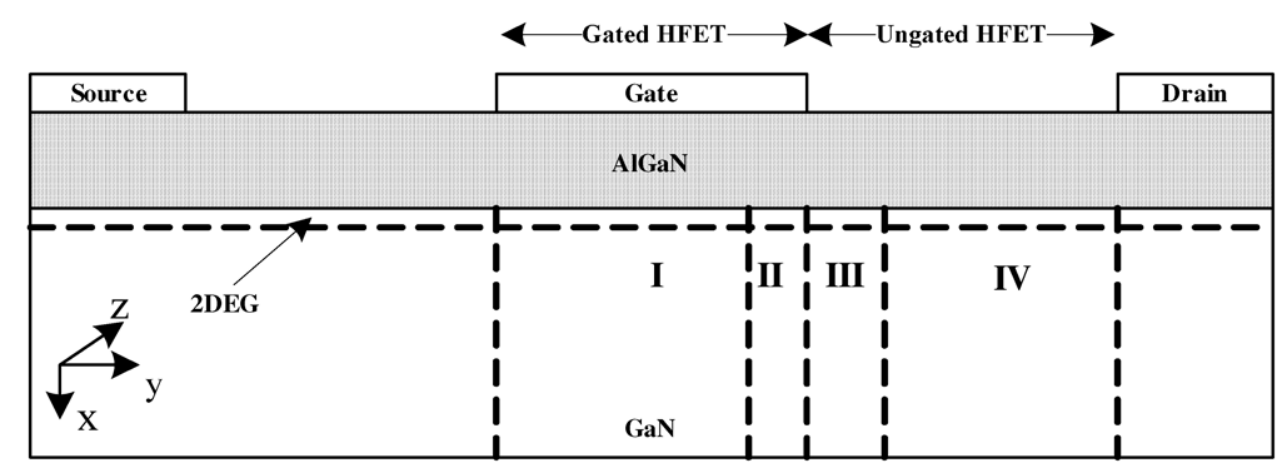

Figure 2. Schematic cross-section of a non-self-aligned AlGaN/GaN HFET. Sections identified by I, II, III and IV are explained in the text. 
Table 1. Parameters used in the simulation.

\begin{tabular}{lll}
\hline Symbol & Description & Value \\
\hline$R_{\mathrm{S}}$ & Source contact resistance & $5 \Omega$ \\
$R_{\mathrm{D}}$ & Drain contact resistance & $5 \Omega$ \\
$L_{\mathrm{GD}}$ & Gate-drain spacing & $1 \mu \mathrm{m}$ \\
$L_{\mathrm{G}}$ & Gate length & $0.25 \mu \mathrm{m}$ \\
$W$ & Gate width & $200 \mu \mathrm{m}$ \\
$V_{\mathrm{T}}$ & Threshold voltage & $-3.75 \mathrm{~V}$ \\
$d$ & Barrier thickness & $17.5 \mathrm{~nm}$ \\
$\Delta d$ & $2 \mathrm{DEG}$ thickness & $2 \mathrm{~nm}$ \\
$\varepsilon_{\mathrm{r}}$ & $\mathrm{Al}_{0.3} \mathrm{Ga}_{0.7} \mathrm{~N}$ relative dielectric constant & 8.78 \\
\hline
\end{tabular}

To obtain the characteristics between the drain-current density and channel potential at the end of region I, for each gate voltage, $J$ is swept from zero to a value above $1 \mathrm{~A} \mathrm{~mm}^{-1}$ in very small increments. Continuity of the electric field along the channel, current continuity in the drift-only channel, and boundary condition of the grounded source electrode are applied to this solution. In solving this equation, longitudinal electric field is monitored. This equation is valid as long as this electric field is smaller than $E_{v-\max }$. The channel potential recorded at the end of region I (i.e. $V_{D-v \text {-max }}$ ) is imported to section 2.1.2 for each value of gate voltage and drain-current density.

2.1.2. Saturation-region characteristics (i.e. $E>E_{v \text {-max }}$ ). As the drain voltage and consequently the magnitude of peak longitudinal electric field increase, early reduction in carrier drift velocity in region II (according to the transport models of (2) and (3)), and eventual saturation of drift velocity observed in all three transport models, causes electron bunching in the latter part of the channel. Considering the track of the seminal work of Grebene and Ghandhi [16], this phenomenon is studied through incorporation of both components of electric field (i.e. the components along the channel and normal to the heterointerface). While the model of Grebene et al only addresses this phenomenon in FETs with purely saturating drift velocity (such as (1)), in this work this model is extended to drift-transport models with steady-state velocity overshoot.

According to the model of Grebene et al, to incorporate the impact of electron bunching in region II, Gauss's law has been applied to the rectangular Gaussian box, shown in figure 3 . This box fully extends throughout region II. In this figure, $X_{j}$ marks the depletion layer thickness in $\mathrm{GaN}$, which is unintentionally doped to $N_{\mathrm{GaN}}$. According to this

$$
\oint \vec{E} \cdot \overrightarrow{\mathrm{d} S}=\frac{Q_{\mathrm{tot}}}{\varepsilon_{\mathrm{s}}}
$$

where $\vec{E}$ is the electric field, $\overrightarrow{\mathrm{d} S}$ is the surface element and $Q_{\text {tot }}$ is the total charge enclosed by the Gaussian box. The absence of a $Z$-directed electric field reduces (9) to a two-dimensional equation:

$$
\begin{gathered}
-\left(\int_{0}^{y^{\prime}} \frac{Q_{n}\left(y^{\prime \prime}\right)}{\varepsilon_{\mathrm{GaN}}} \mathrm{d} y^{\prime \prime}+\frac{q N_{\mathrm{GaN}} X_{j}}{\varepsilon_{\mathrm{GaN}}} y^{\prime}\right)=-E_{y}\left(y^{\prime}\right) X_{j} \\
+E_{v-\max } X_{j}-\frac{\varepsilon_{\mathrm{AlGaN}}}{\varepsilon_{\mathrm{GaN}}} \int_{0}^{y^{\prime}} E_{\perp}\left(0, y^{\prime \prime}\right) \mathrm{d} y^{\prime \prime} .
\end{gathered}
$$

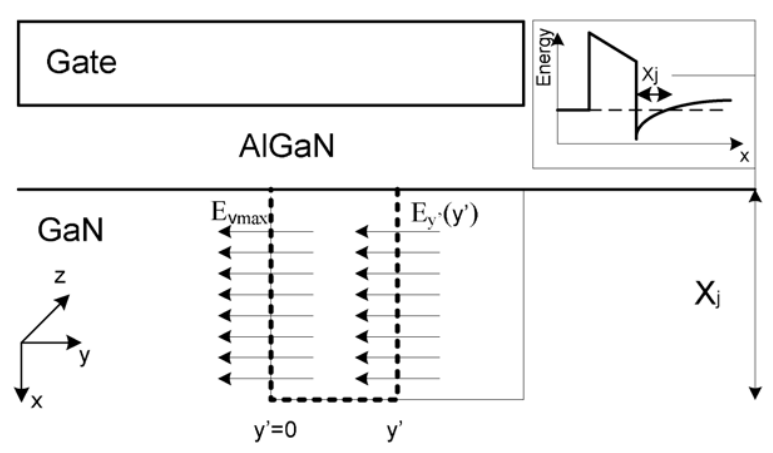

Figure 3. Schematic cross-section of a self-aligned AlGaN/GaN HFET, illustrating the Gaussian box used in the analysis of the saturation region. The inset shows the conduction band edge at the $\mathrm{AlGaN} / \mathrm{GaN}$ heterointerface.

The normal electric field to $\mathrm{AlGaN} / \mathrm{GaN}$ heterointerface (i.e. $E_{\perp}$ ) is given by

$$
E_{\perp}\left(0, y^{\prime}\right)=\frac{1}{d+\Delta d}\left(V_{\mathrm{GT}}+\frac{Q_{\mathrm{B}}}{C_{\mathrm{ch}}}-V\left(y^{\prime}\right)\right)
$$

where $Q_{\mathrm{B}}$ is the total bulk charge in the $\mathrm{GaN}$ region enclosed by the Gaussian box.

$Q_{n}$ in (10) is the sheet-charge density and unlike the assumption of Grebene et al for the transport models such as (2) and (3) it cannot be assumed to be constant:

$$
Q_{n}\left(y^{\prime}\right) \simeq C_{\mathrm{ch}}\left(V_{\mathrm{GT}}^{\prime}-V\left(y^{\prime}\right)\right)
$$

In this form, the position dependence of the sheet-charge density along the length of region II is incorporated through the variation of channel potential (i.e. $\left.V\left(y^{\prime}\right)\right)$ and the threshold voltage $V_{\mathrm{T}}^{\prime}\left(y^{\prime}\right)$. In this equation, $V_{\mathrm{GT}}^{\prime}$ is defined as $V_{\mathrm{G}}-V_{\mathrm{T}}^{\prime}\left(y^{\prime}\right)$. In the model of Grebene et al, the constant profile of sheetcharge density in region II has been maintained by assuming the same position dependence for the threshold voltage and channel potential. Although this is correct for the saturating $v-E$ profile of (1), it will not be acceptable for the transport models such as (2) and (3). As is explained later in this section, the profile of threshold voltage will be calculated through iteratively solving (10) in pair with the current-continuity equation. In this calculation, due to the existence of a nonzero gradient in electron concentration along the length of region II, the assumption of a drift-only channel ceases to be sufficient. As a result, for each value of drain current the amount of diffusion current, calculated through the evaluation of this gradient, should be subtracted to yield the drift-current component required by the aforementioned equations.

By inserting (11) and (12) in (10), the following relationship between the device threshold voltage (i.e. $V_{T}$ ) and the position-dependent effective threshold voltage of region II (i.e. $\left.V_{\mathrm{T}}^{\prime}\left(y^{\prime}\right)\right)$ is achieved:

$$
X_{j} \frac{\partial E\left(y^{\prime}\right)}{\partial y^{\prime}}=-\frac{C_{\mathrm{ch}}}{\varepsilon_{\mathrm{GaN}}}\left(V_{\mathrm{T}}-V_{\mathrm{T}}^{\prime}\left(y^{\prime}\right)\right) .
$$

In solving for the position dependence of the threshold voltage in region II, the channel potential and electric-field 
distributions of [16] can be taken as initial estimates:

$$
\begin{gathered}
E\left(y^{\prime}\right)=E_{v-\max } \cosh \left(\frac{y^{\prime}}{\lambda}\right) \\
V\left(y^{\prime}\right)=V_{D-v-\max }+\lambda E_{v-\max } \sinh \left(\frac{y^{\prime}}{\lambda}\right) .
\end{gathered}
$$

In which, $V_{D-v \text {-max }}$ is the channel potential at the end of region I. In this work, the initial value of the parameter $\lambda$ is taken equal to $30 \mathrm{~nm}$, which is in the range of values reported in the literature [9]. Adoption of these initial estimates to (12) will result in an initial estimate for $V_{\mathrm{T}}^{\prime}\left(y^{\prime}\right)$, which according to (11) will result in a position-dependent profile for $Q_{n}\left(y^{\prime}\right)$. The profile of sheet-charge density in region II is then used in calculation of the diffusion-current component in this region. By modifying the drift-current component for any given value of drain current, a new profile for the effective threshold voltage is then calculated:

$$
\begin{gathered}
J_{\mathrm{drift}}=J-J_{\mathrm{diff}} \\
V_{\mathrm{T}}^{\prime}\left(y^{\prime}\right)=V_{\mathrm{G}}-V\left(y^{\prime}\right)-\frac{J_{\mathrm{drift}}\left(y^{\prime}\right)}{C_{\mathrm{ch}} v\left(y^{\prime}\right)} .
\end{gathered}
$$

Plugging this new profile of effective threshold voltage in (13) results in revised profiles for the channel potential and electric field along the length of region II. Continuity of electric field should be maintained at the boundary of regions I and II. For every value of gate voltage (i.e. $V_{\mathrm{G}}$ ) and drain current (i.e. $J$ ) this procedure is repeated until convergence. The aforementioned correction of the drift-current component is applicable to that portion of region II in which $E\left(y^{\prime}\right)$ is smaller than the velocity-saturation electric field. Beyond this point, assumptions of Grebene et al in the form of (13) and (14) are valid. A flowchart of this procedure is provided in figure 4 .

\subsection{Drain-current simulation of non-self-aligned HFET}

The ungated HFET channel of the drain-access region, shown in figure 2, in resemblance to the gated HFET can be broken into a linear and a saturation region. In figure 2 , these two regions are marked as regions IV and III, respectively.

While the relationship between the current and voltage at the drain edge of the gate electrode, for each value of gate voltage, can be calculated according to the procedure explained in section 2.1, the potential drop across the drainaccess region is accounted for by implementing a procedure very close to that of figure 4 . The only remarkable difference between the gated and ungated HFET is that, in this region in the absence of a metallic gate, a surface-potential term should instead be considered. In support of this argument, it should be recalled that the theory of metal semiconductor junctions, due to lack of consideration of surface states, has the tendency to overestimate the role of metal work function. The current continuity and continuity of electric field between regions II and III should be maintained. In the absence of an actual gate voltage, the role of the apparent spatial variation of the surface potential can be lumped into the variation of the effective threshold voltage. By doing so, no information is lost.

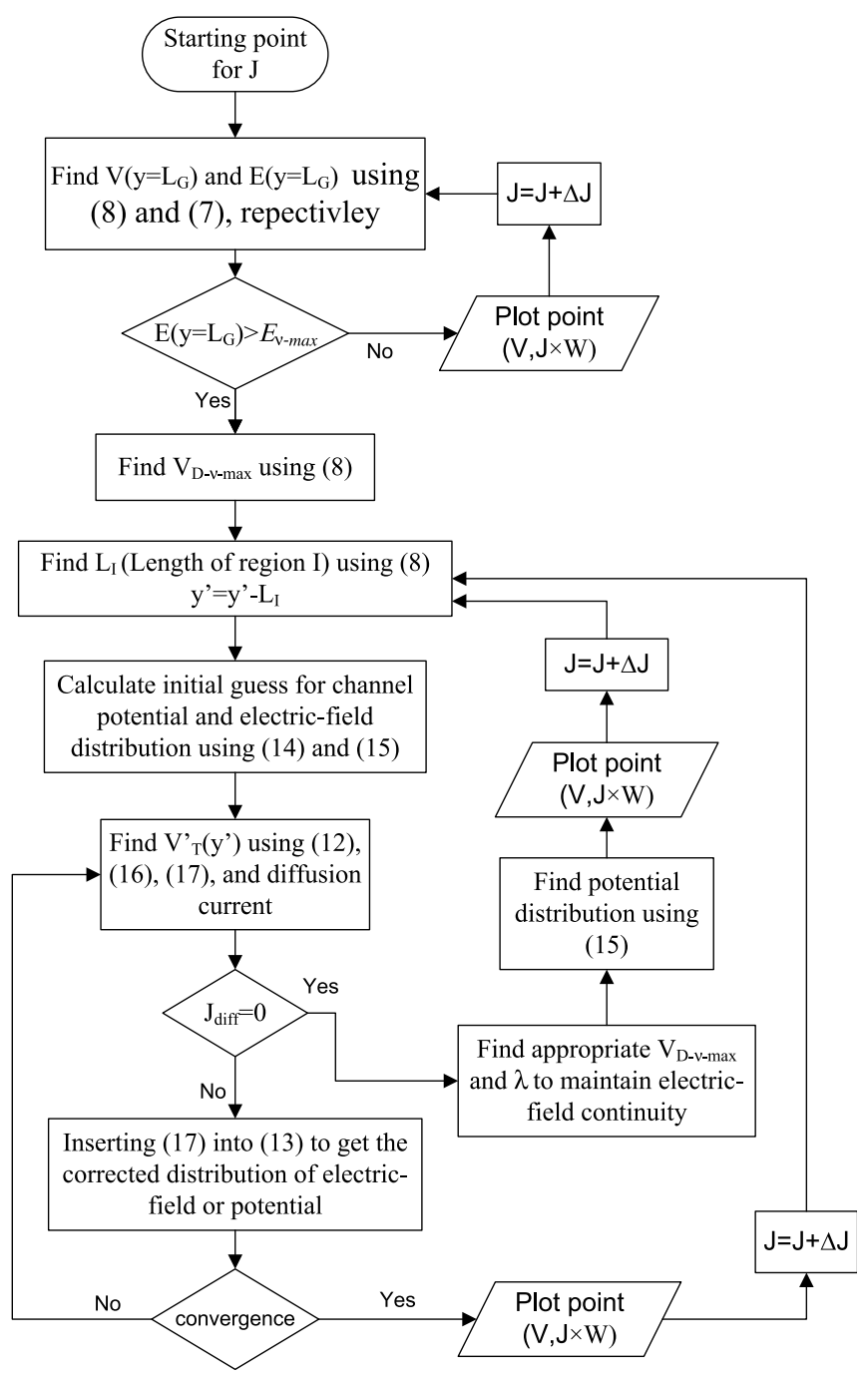

Figure 4. Simulation flowchart for the self-aligned AlGaN/GaN HFET.

This is because in the calculation of charge concentration along the channel, the difference between $V_{\mathrm{G}}\left(y^{\prime}\right)$ and $V_{\mathrm{T}}^{\prime}\left(y^{\prime}\right)$ and not their individual values is of interest. The value of the constant part of the surface potential (used in place of $V_{\mathrm{G}}$ in equations of section 2) is set by sweeping $V_{\mathrm{G}}$ and finding the proper value for which the electric-field continuity is maintained at the boundary of regions II and III.

\section{Simulation results and discussions}

Figure 5 illustrates examples of convergence of threshold voltage, diffusion-current density and electric field in the saturation region of the gated channel (i.e. region II). Acceptable levels of convergence after 6 steps of iteration have been observed to reduce the burden of choosing the fitting parameter (e.g. $\lambda$ ).

Figures $6(a),(b),(c)$ and $(d)$ demonstrate the drain current-voltage characteristics of AlGaN/GaN HFETs of identical structural parameters with the assumption of drain and source contact resistances of $0 \Omega, 5 \Omega, 20 \Omega$ and $35 \Omega$, respectively. These figures illustrate a comparison between 

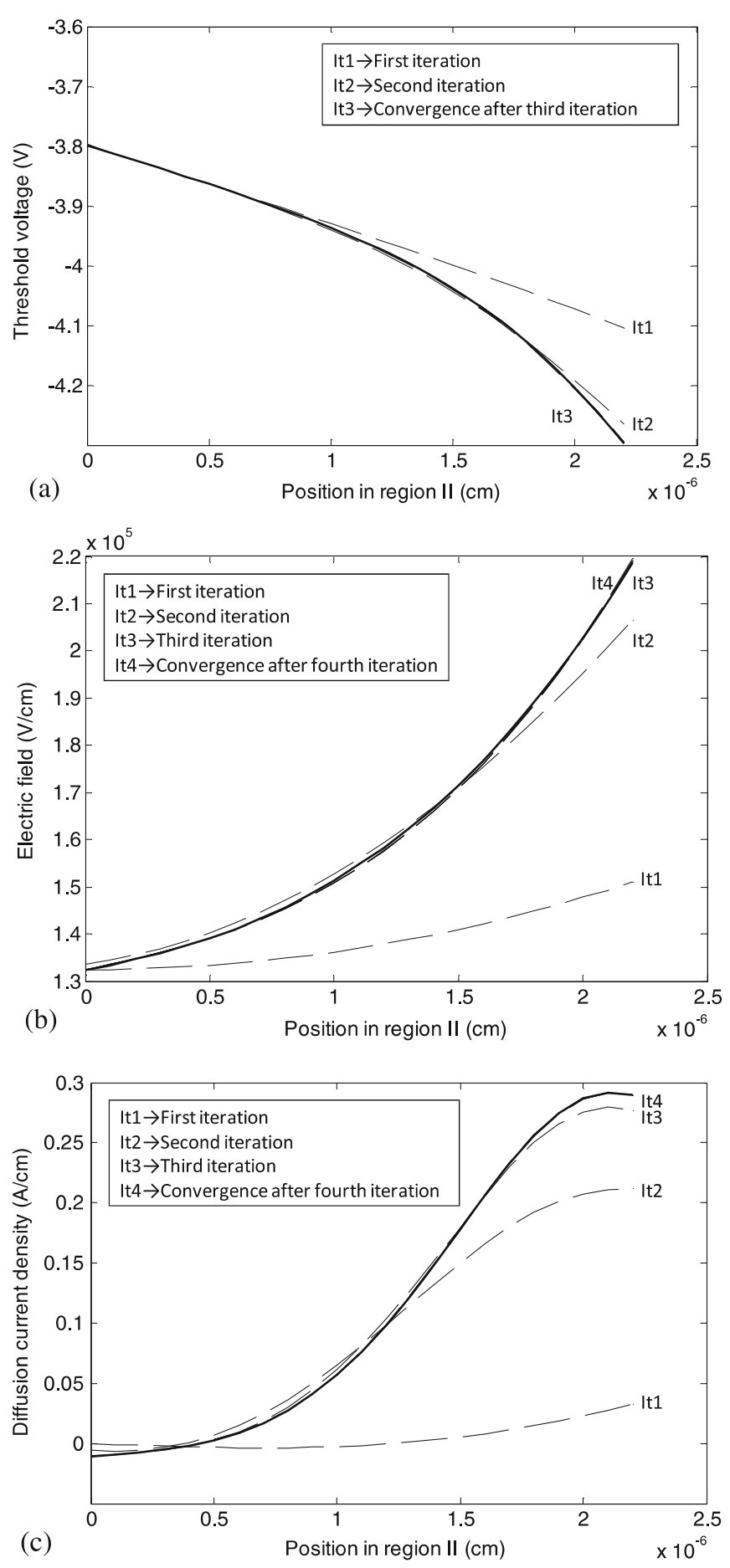

Figure 5. Convergence of threshold voltage distribution $(a)$, electric-field distribution $(b)$ and diffusion-current density $(c)$ in region II after a few steps of iteration for $V_{\mathrm{G}}=-1.8 \mathrm{~V}, I=124 \mathrm{~mA}$. Convergence to the curves (solid) is achieved. Iteration numbers are indicated on each curve. In spite of convergence after fewer iteration steps than six, six iteration steps have been performed to validate the complete convergence of the characteristics.

the results of the adoption of transport models of (1), (2) and (3). Observation of the variations of drain currentvoltage characteristics with contact resistance shows that in devices with higher contact resistance, the impact of steady-state drift-velocity overshoot is considerably masked.
Through comparison between the simulation results of the three transport models, it is projected that with improvement of Ohmic contact technology the broad velocity-overshoot pattern, predicted by the more accurate transport model of (2), can provide a substantially higher drain-current level. The differences between the transport models of (2) and (3), which are shown in figure 1 , indicate that the wider overshoot pattern and higher low-field electron mobility predicted by (2) provide a higher saturation drain current for any value of gate-source voltage.

Figure 7, illustrates the match between the results of simulation based on the assumption of Ridley saturating transport model of (1), and a set of experimental drain current-voltage characteristics. Contact resistance of $2 \Omega$ has been assumed in the simulation. Measurements have been performed on an AlGaN/GaN HFET grown by molecular beam epitaxy (MBE) on a SiC substrate. The device has two gate fingers with a gate length of $0.25 \mu \mathrm{m}$ and a gate finger width of $100 \mu \mathrm{m}$. The Hall electron mobility of this sample was reported to be about $1000 \mathrm{~cm}^{2} \mathrm{~V}^{-1} \mathrm{~s}^{-1}$. As was indicated earlier, this value of low-field electron mobility has been considered in choosing the parameters of the three transport models.

According to the trends observed in figure 6 , the match between the experimental data and the simulation with the adoption of transport model of (1) and low contact resistance indicates the presence of high contact resistance, in the order of less than $20 \Omega$, in the measured device. This is because the more accurate transport model of (2), for this range of contact resistance, predicts saturation drain-current values close to the experiments (also shown in figure 7). As shown in figure 6, for this range of contact resistance, assumption of a saturating transport model will not produce accurate insight into the device.

The more pronounced role of larger electric fields in devices of shorter gate length predicts that the draincurrent gain from the increased width of drift-velocity overshoot pattern, between transport models of (2) and (3), should be smaller in these devices. This is because both of these models are predicting the same values of drift velocity for moderate to high electric fields. In support of this argument, figure 8 illustrates drain current-voltage characteristics for three values of gate length (i.e. $0.25,0.5$ and $1 \mu \mathrm{m})$, assuming that all other device parameters are kept identical.

These observations indicate that in contrast to the traditional assumptions of negligible impact of steady-state velocity overshoot on drain-current characteristics of III-V HFETs, for AlGaN/GaN HFETs the exact nature of the drift transport can have very substantial impact on the drain current among other characteristics. This is due to the broader velocity-overshoot pattern and also larger value of electric fields normally applied to these wide-bandgap HFETs. Despite the fact that these features are partially masked by parasitic effects such as contact resistance in the current state of the art of this technology, with the constant technological improvement of III-nitride HFETs [11,17], a more complete inclusion of transport characteristics in the fashion presented in this paper becomes a necessity. 

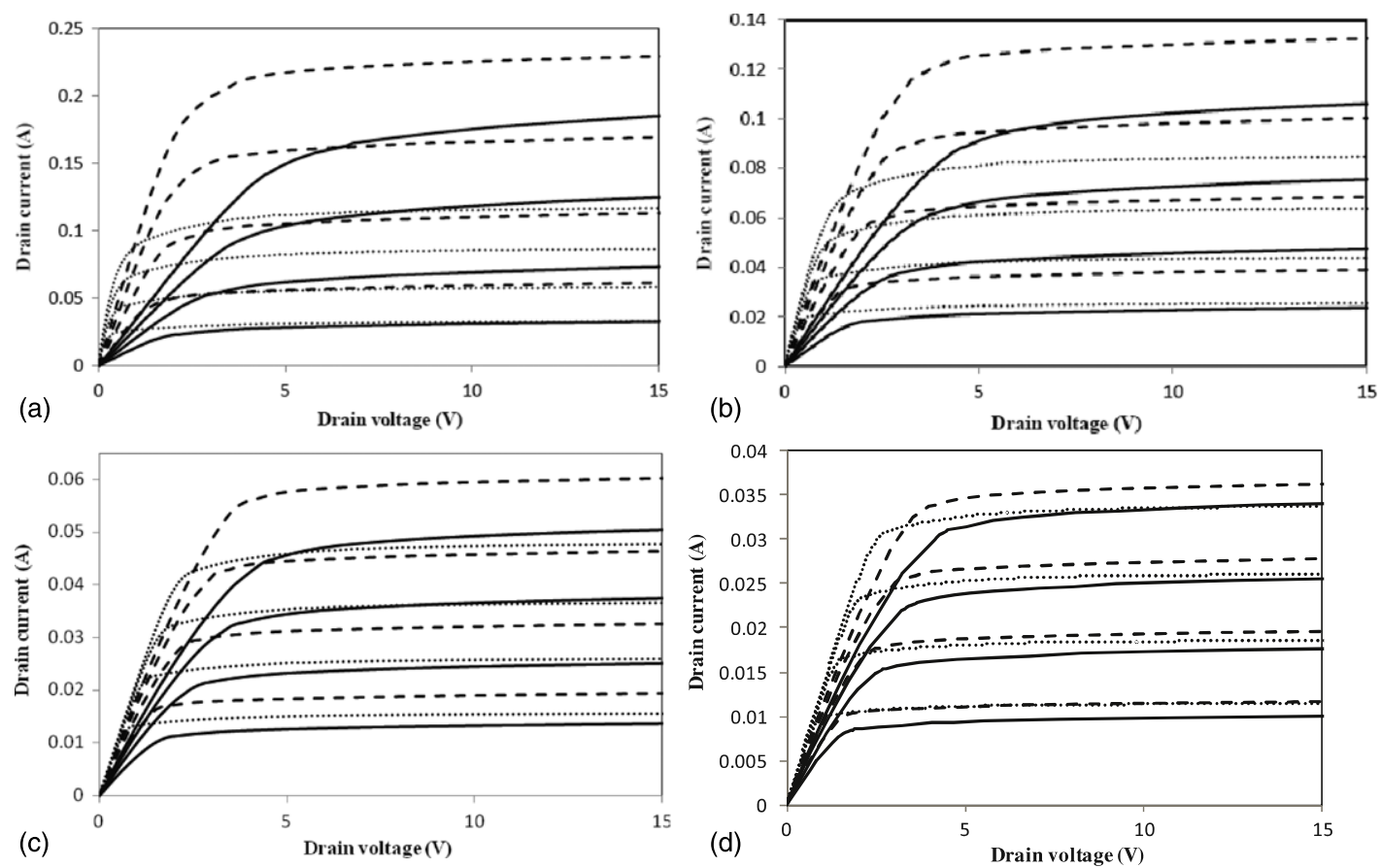

Figure 6. Drain $I-V$ characteristics based on mobility models of (1), (2), (3), shown with dotted curves, discontinuous curves and solid curves, respectively. Each part includes the data for $V_{\mathrm{G}}=-1.8$ to $-3.0 \mathrm{~V}$ with steps of $0.4 \mathrm{~V}$. In $(a)$ data are presented for $R_{\mathrm{S}}$ and $R_{\mathrm{D}}$ equal to $0 \Omega$. In $(b)$ data are presented for $R_{\mathrm{S}}$ and $R_{\mathrm{D}}$ equal to $5 \Omega$. In $(c)$ data are presented for $R_{\mathrm{S}}$ and $R_{\mathrm{D}}$ equal to $20 \Omega$. In $(d)$ data are presented for $R_{\mathrm{S}}$ and $R_{\mathrm{D}}$ equal to $35 \Omega$.

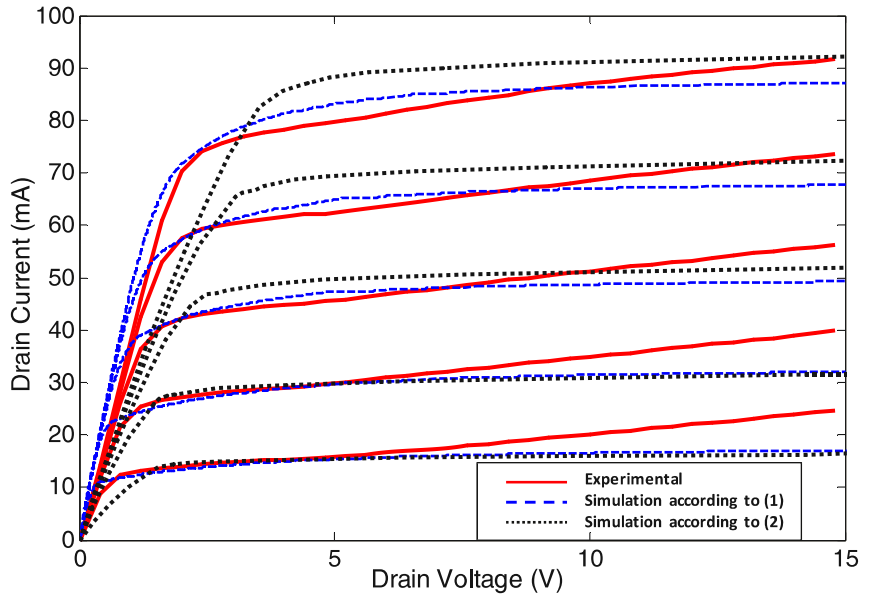

Figure 7. Drain $I-V$ characteristics based on mobility models (1) and (2) (shown in dashed and dotted curves, respectively) versus experimental measurements (solid curve) for $V_{\mathrm{G}}=-1.8$ to $-3.4 \mathrm{~V}$ with steps of $0.4 \mathrm{~V}$. In simulation with mobility model (1), the device is a non-self-aligned HFET with $R_{\mathrm{S}}$ and $R_{\mathrm{D}}$ of $2 \Omega$, and $L_{\mathrm{GD}}=1.875 \mu \mathrm{m}$. In simulation with mobility model (2), only $R_{\mathrm{S}}$ and $R_{\mathrm{D}}$ were changed to $20 \Omega$. The mismatch close to threshold voltage is due to the lack of consideration of leakage through the buffer layer.

(This figure is in colour only in the electronic version)

In this one-dimensional investigation, the effect of heating has not been considered. Due to the application of small drain voltages and also the presence of highly heat-conductive $\mathrm{SiC}$ substrate the experimental data also do not show any thermal effect. It should also be noted that due to the application of a voltage between drain and source terminals several factors including the width of the quantum well, sub-band energy levels, sheet carrier concentration and confinement of electron wave at the heterojunction are prone to change along the channel. Such effects are not usually considered in analytical modelling of the drain-current characteristics of HFETs for moderate drain voltages [9]. In order to reduce the complexity of the presented model, these effects are not considered here. It is believed that for the moderate drain voltages, this is an acceptable approximation. Lack of inclusion of these factors is expected to be partially responsible for the larger output resistance predicted in the simulated curves with regards to the experimental data.

\section{Conclusion}

An analytical model for drain-current characteristic of AlGaN/GaN HFETs with incorporation of inflection points and steady-state velocity overshoot is proposed. The application of this model to HFETs reveals the tangible impact of these features on drain-current characteristics. The presented model, which is based on iterative solution of current continuity and Poisson's equation, is quite independent of the choice of fitting parameters. It is shown that by technological improvement of the Ohmic contacts in III-nitride technology, the effects of transport features such as inflection points and steady-state velocity overshoot gain further relevance in producing highly reliable models for these devices. It has been observed that the inflection points of drift-transport characteristics will play a prominent role in enhancing the drain-current level of these devices. 

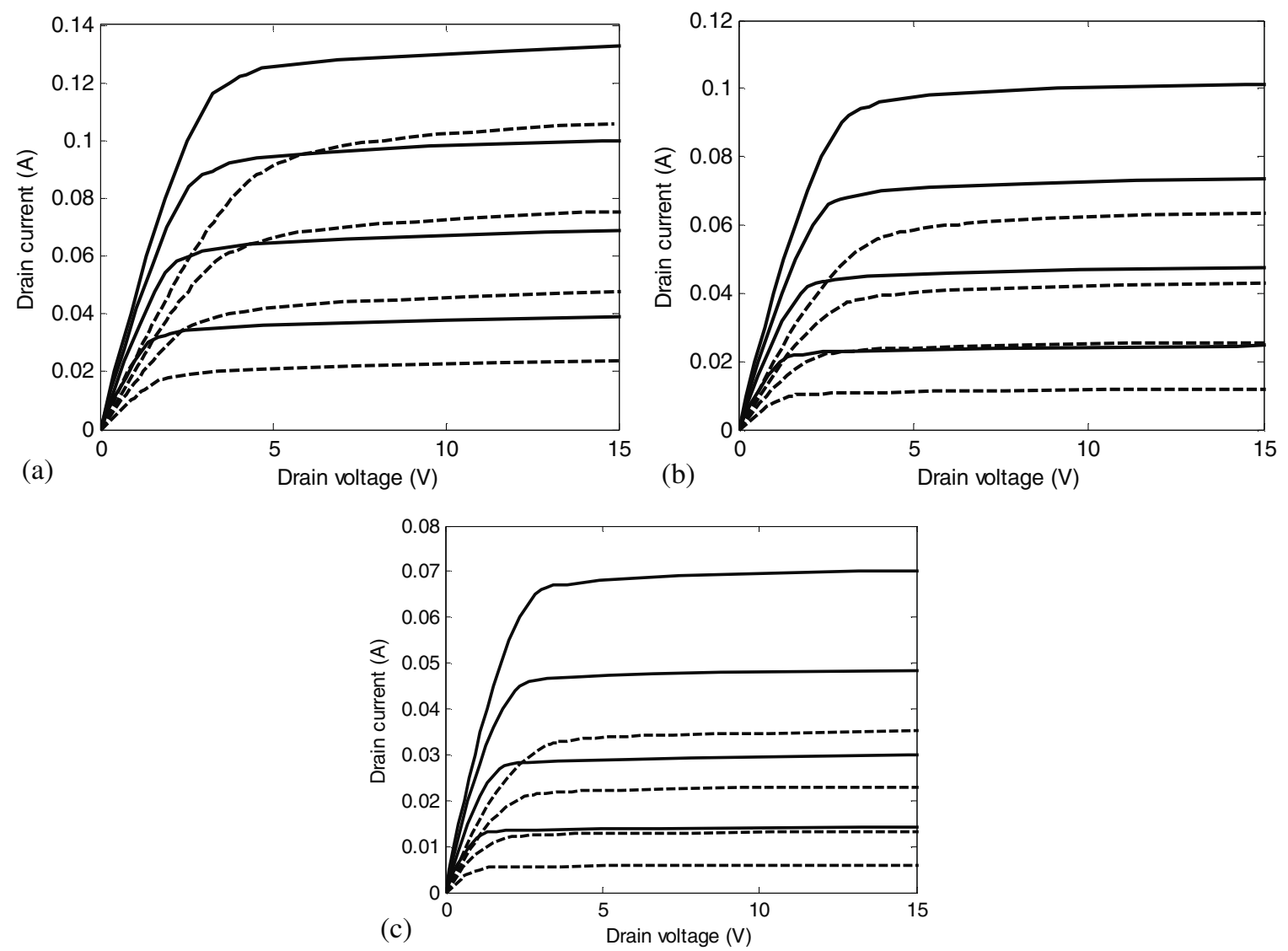

Figure 8. Drain $I-V$ characteristics based on transport model of (2) (solid line), versus (3) (dashed line) for $L_{\mathrm{G}}=0.25 \mu \mathrm{m}(a)$ $L_{\mathrm{G}}=0.5 \mu \mathrm{m}(b)$ and $L_{\mathrm{G}}=1 \mu \mathrm{m}(c)$, for $V_{\mathrm{G}}=-1.8$ to $-3.0 \mathrm{~V}$ with steps of $0.4 \mathrm{~V}$. $R_{\mathrm{S}}$ and $R_{\mathrm{D}}$ are equal to $5 \Omega$.

\section{References}

[1] Fujito K, Kubo S and Fujimura I 2009 Development of bulk GaN crystals and nonpolar/semipolar substrates by HVPE MRS Bull. 34 313-7

[2] Dwilinski R, Doradzinski R, Garczynski J, Sierzputowski L, Puchalski A, Kanbara Y, Yagi K, Minakuchi H and Hayashi H 2009 Bulk ammonothermal GaN J. Cryst. Growth 311 3015-8

[3] Lin H, Yu H and Huang F 2010 Performance improvement of AlGaN/GaN HEMTs using two-step silicon nitride passivation Microw. Opt. Technol. Lett. 52 1614-9

[4] Heller E 2008 Simulation of life testing procedures for estimating long-term degradation and lifetime of AlGaN/GaN HEMTs IEEE Trans. Electron Dev. $552554-60$

[5] Bhapkar U and Shur M 1997 Monte Carlo calculation of velocity-field characteristics of wurtzite GaN J. Appl. Phys. 82 1649-55

[6] Shey A and Ku W 1989 An analytical current-voltage characteristics model for high electron mobility transistors based on nonlinear charge-control formulation IEEE Trans. Electron Dev. 36 2299-306

[7] Ridley B 2001 Coupled surface and channel transport in semiconductor heterostructures J. Appl. Phys. 90 6135-9

[8] Polyakov V and Schwierz F 2001 Influence of electron mobility modeling on DC $I-V$ characteristics of WZ-GaN MESFETs IEEE Trans. Electron Dev. 48 512-6

[9] Koudymov A, Shur M, Simin G, Chu K, Chao P, Lee C, Jimenez J and Balistreri A 2008 Analytical HFET I-V model in presence of current collapse IEEE Trans. Electron Dev. 55 712-20

[10] Feng Q, Li L, Hao Y, Ni J and Zhang J 2009 The improvement of ohmic contact of Ti/Al/Ni/Au to AlGaN/GaN HEMT by multi-step annealing method Solid-State Electron. 53 955-8

[11] Mohammed F, Wang L, Koo H and Adesida I 2007 Anatomy-performance correlation in Ti-based contact metallizations on $\mathrm{AlGaN} / \mathrm{GaN}$ heterostructures J. Appl. Phys. 101033708

[12] Kolnik J, Oguzman I, Brennan K, Wang R, Ruden P and Wang Y 1995 Electron transport studies of bulk zinc blende and wurtzite phases of $\mathrm{GaN}$ based on ensemble Monte Carlo calculation including a full zone band structure $J$. Appl. Phys. 78 1033-8

[13] Albrecht J, Wang R, Ruden P, Farahmand M and Brennan K 1998 Electron transport characteristics of $\mathrm{GaN}$ for high temperature device modeling J. Appl. Phys. 83 4777-81

[14] O’Leary S, Foutz B, Shur M and Eastman L 2006 Steady-state and transient electron transport within the III-V nitride semiconductors, $\mathrm{GaN}$, AlN, and $\mathrm{InN}$ : a review J. Mater. Sci.: Mater. Electron. 17 87-126

[15] Morkoç H 2009 Handbook of Nitride Semiconductors and Devices vol 3 Physics and Technology of GaN Based Optical and Electronic Devices (Weinheim: Wiley)

[16] Grebene A and Ghandhi S 1969 General theory for pinched operation of the junction-gate FET Solid-State Electron. 12 573-89

[17] Chung J, Piner E and Palacios T 2009 N-face GaN/AlGaN HEMTs fabricated through layer transfer technology IEEE Electron Dev. Lett. 30 113-6 